\title{
NECTAR YEASTS IN DELPHINIUM NUTTALLIANUM (RANUNCULACEAE) AND THEIR EFFECTS ON NECTAR QUALITY
}

Authors: Robert N. Schaeffer ${ }^{1,2,5}$, Rachel L. Vannette ${ }^{3}$, and Rebecca E. Irwin ${ }^{1,2,4}$

\section{Author Affiliations:}

${ }^{1}$ Department of Biological Sciences, Dartmouth College, Hanover, NH 03755

${ }^{2}$ Rocky Mountain Biological Laboratory, Gothic, CO 81224

${ }^{3}$ Department of Biology, Stanford University, Stanford, CA 94305

${ }^{4}$ Current address: Department of Applied Ecology, North Carolina State University, Raleigh, NC 27695

${ }^{5}$ Corresponding Author and current address: Robert N. Schaeffer, Department of Biology, Tufts University, 163 Packard Ave., Medford, MA 02155; Robert.Schaeffer@Tufts.edu

Running Head: Yeasts in Delphinium nectar

Article history:

Received 20 March 2015

Revision received 22 July 2015

Accepted 29 August 2015

Corresponding editor:

Anne Pringle 


\begin{abstract}
Microorganisms colonize the nectar of many angiosperms. Variable diversity and spatiotemporal dynamics of nectar-inhabiting microorganisms (e.g., yeasts) may drive variation in nectar sugar composition and subsequent plant-pollinator interactions. We assessed yeast frequency of occurrence and density in the nectar of the perennial herb, Delphinium nuttallianum, across multiple spatio-temporal scales, including flower lifetime and sex-phase transition, flowering season, populations, and years. We tested the hypothesis that pollinators vector yeasts by comparing densities between virgin flowers and those open to visitation. Finally, we identified yeasts using molecular methods and tested for an association between yeast density and nectar composition using ultra-performance liquid chromatography. Yeasts were frequent colonists of Delphinium nectar, occurring in all populations and years sampled. Yeast frequency of occurrence and density varied across most spatio-temporal scales examined. Pollinators were vectors of yeast: virgin flowers remained yeast-free, while those open to visitation became inoculated. Nectar samples were species-poor, with a majority colonized by Metschnikowia reukaufii. Finally, increasing yeast density was correlated with a decrease in sucrose and an increase in monosaccharides. Our results document that yeasts form species-poor communities in populations of this hermaphroditic perennial, in addition to highlighting their spatio-temporal dynamics and effects on nectar quality. Spatio-temporal variation in frequency
\end{abstract}


of occurrence, density, and changes in nectar may have important implications for the nature and strength of interactions between Delphinium and its pollinators.

Keywords: Delphinium nuttallianum, floral nectar, Metschnikowia reukaufii, nectar composition, nectar yeasts, pollination 


\section{Introduction}

Floral nectar is a resource important for meditating interactions between plants and animals (Simpson and Neff, 1983). Traditionally viewed as a reward for mutualist floral visitors in exchange for pollination services conferred, nectar has been the focus of considerable attention, including research to understand the links between its nutritional and chemical content and floral visitor identity and behavior (Baker and Baker, 1973, 1983, 1987). From a microbiological perspective, floral nectar can also serve as habitat for microorganisms such as yeasts and bacteria that are vectored amongst plants by flower visitors (Brysch-Herzberg, 2004; Herrera et al., 2008; Álvarez-Pérez et al., 2012; Fridman et al., 2012).

Yeasts are commonly found in the nectar of angiosperms species (Brysch-Herzberg, 2004; Herrera et al., 2008; Belisle et al., 2012; Canto and Herrera, 2012). Evidence suggests that their frequency of occurrence and potential to modify floral nectar rewards make them key third-party interactors in plant-pollinator communities, with the potential to affect the strength of plantpollinator mutualisms through both direct and pollinator-mediated indirect mechanisms (Herrera et al., 2008, 2013; Schaeffer and Irwin, 2014). Yeast metabolic activity may affect floral nectar traits, such as nectar temperature (Herrera and Pozo, 2010), scent (Raguso, 2004; Goodrich et al., 2006; Golonka et al., 2014), and amino acid content (Kevan et al., 1988; Peay et al., 2012).

Moreover, yeast activity may reduce nectar sugar concentrations and alter sugar ratios (Herrera et al., 2008; Canto and Herrera, 2012). Despite the supposition that nectar yeasts may alter nectar sugar rewards, there are still surprisingly few documented cases of the relationship between nectar yeasts and nectar sugar composition (de Vega and Herrera, 2012; Golonka and Vilgalys, 2013; Pozo et al., 2014), and strong effects of yeasts on sugar composition may not be universal (Peay et al., 2012). 
Nectar yeasts are viewed as ubiquitous, and surveys of nectar microbial dynamics, especially spatio-temporal dynamics, and diversity are growing in number (but see Herrera et al., 2009; Belisle et al., 2012; de Vega and Herrera, 2012). Of the studies that have been conducted thus far, significant spatio-temporal variation in patterns of yeast occurrence has been observed. For example, within a Mimulus aurantiacus population, Belisle et al. (2012) found that host plant spatial location, in addition to flower density, influenced patterns of yeast occurrence. Moreover, Herrera et al. (2009) found that plant communities in different regions of Spain and Mexico varied in the frequency of yeast occurrence and densities reached, likely a function of interspecific differences amongst host plant species in nectar quality and their associated pollinator visitors. Furthermore, patterns of yeast occurrence and density can vary across numerous temporal scales, including flower lifetime (Herrera et al., 2008; Peay et al., 2012), within flowering seasons (Pozo et al., 2014), and across flowering seasons (Golonka and Vilgalys, 2013). Turning to nectar yeast diversity, one common pattern emerging from surveys is low species richness of nectar yeasts, potentially due to nectar filtering of the yeast community (e.g., Herrera et al., 2010) or strong priority effects in nectar (e.g., Peay et al., 2012; Vannette and Fukami, 2014). More studies are needed to assess whether the generality of these patterns hold across other plant species.

Here, we explored the diversity and spatio-temporal dynamics of nectar yeasts associated with the montane perennial larkspur Delphinium nuttallianum (Ranunculaceae; hereafter Delphinium), and their effects on nectar sugar composition. Numerous members of the Ranunculaceae have been found to harbor yeasts in their nectar (Brysch-Herzberg, 2004; Herrera et al., 2008), including Delphinium (Schaeffer and Irwin, 2014). Variation in nectar rewards exists amongst Delphinium individuals in natural populations (Pleasants and Zimmerman, 1979; 
Waser and Mitchell, 1990). Variation in nectar yeasts among flowers and plants could, in part, play a role in creating variation in total amount of sugar as well as sugar composition, with implications for the evolutionary ecology of plant-pollinator interactions. In this study, we investigated: (i) the frequency of occurrence, density, and identity of yeasts in the floral nectar of Delphinium; (ii) how they vary spatio-temporally in frequency of occurrence and density amongst Delphinium, at scales including flower lifetime, within a flowering season, and across populations and years; and (iii) the relationship between yeast density and variation in nectar sugar components. Variation in yeast occurrence and density, coupled with effects on nectar traits, may have significant consequences for plant-pollinator interactions and resulting plant fitness.

\section{Materials and methods}

\section{Study areas and system}

This study was conducted in three populations of the montane larkspur Delphinium nuttallianum (Ranunculaceae), near the Rocky Mountain Biological Laboratory (RMBL), in the Elk Mountains of central Colorado, USA from 2009-2013. Nectar was sampled from Delphinium flowers in populations at Brush Creek (BC: 3853'45.94"N, 10653'18.34"W), Deer Creek (DC: 38 56'48.34" N, 106 58'46.86" W), and Spring Creek (SC: 3851'53.36" N, 106³9'49.22" W). Around RMBL, Delphinium is a short-lived perennial that flowers from late May to early July. The nectar-spurred, purple flowers of Delphinium are protandrous, zygomorphic, and last approximately $6 \mathrm{~d}$, each producing $0.5-1.5 \mu \mathrm{l}$ of nectar over $24 \mathrm{hr}$ with $51.2 \pm 0.7 \%$ (mean $\pm \mathrm{SE})$ sugar concentration (Waser, 1978). Individual plants typically produce a single raceme with 1-15 flower buds that mature in a bottom-up fashion. Delphinium flowers are weakly self-compatible 
(Price and Waser, 1979); however, protrandry prevents autogamous self-pollination and very few seeds are produced in the absence of pollinator visitation (Waser, 1978). Thus, pollinators are required to transfer even self-pollen within flowers and plants (Waser and Price, 1991), and seed set is often limited by pollinator visitation, with greater visitation increasing stigma pollen loads and seed set as a saturating function (Bosch and Waser, 1999). The most common flower visitors to Delphinium around RMBL are queen bumble bees (Bombus appositus, B. balteatus, B. californicus, B. flavifrons, B. frigidus, B. nevadensis (Apidae)) and one hummingbird, Selasphorus platycercus (Trochilidae) (Waser, 1978; Waser and Price, 1981, 1991).

\section{Nectar sampling}

To assess the frequency of yeast occurrence in Delphinium nectar and their density, in all years and at all sites, nectar samples were collected using the following approach. Plants with open flowers were bagged using fine-mesh bags constructed of bridal veil at the whole plant level. Flowers that were open prior to bagging were marked and collected $24 \mathrm{hr}$ later; a time that allows for sufficient nectar to accumulate for sampling. Being open prior to bagging, these flowers were exposed to pollinators and had the potential to be inoculated with yeasts (BryschHerzberg, 2004; Herrera et al., 2008, 2009). Upon collection, flowers were stored in individual, new zip-loc bags, placed in a cooler, and returned to the laboratory for processing of nectar samples. Unless noted otherwise, a single flower was collected per plant per sampling event, and zip-lock bags were never re-used.

The following bagging schemes were used to determine how the frequency of yeast occurrence and density varied across multiple spatial and temporal scales. To confirm that floral visitors vector yeasts and inoculate flowers, in 2009 on June 1830 plants were randomly bagged 
at DC with virgin flowers still in the bud stage. By doing so, flowers were prevented from being visited by pollinators or other insects that may vector yeasts. A second set of 30 plants with open flowers was also bagged. Open, female-phase flowers of approximately similar age ( $\sim 5-6 \mathrm{~d}$ old $)$ were collected from both sets of plants for comparison.

The following approaches were then used to assess temporal and/or spatial variation in yeast occurrence and density. First, at the scale of individual flowers, in 201230 plants were bagged each at BC (May 17), DC (May 28), and SC (June 13) when populations were near peak flower abundance, marking an open male- ( $\sim 2-3 \mathrm{~d}$ old $)$ and female-phase $(\sim 5-6 \mathrm{~d}$ old $)$ flower on each individual plant for collection. Characterization of differences between sex phases allowed us to determine how yeast densities varied with flower age, as Delphinium is protandrous and transitions to female-phase as the flower matures. This sampling scheme also allowed us to test for spatial variation in the degree of yeast occurrence and densities across populations. Second, to assess temporal variation within a season, at BC 20 plants were randomly bagged every other day from May 11-27 throughout the course of the 2012 flowering season. To examine how yeast densities may be influenced by flower density within a season, flower density was estimated on each sampling date using the following approach. Two bisecting $25 \mathrm{~m}$ transects were established across the sampling area and every $5 \mathrm{~m}$ along each transect, all flowers on each individual Delphinium were counted within a $1 \mathrm{~m}^{2}$ quadrat. Third, to assess spatial variation across populations, nectar was collected from additional bagged plants at $\mathrm{SC}(\mathrm{N}=30)$ at peak floral abundance for comparison with samples obtained at $\mathrm{BC}(\mathrm{N}=20)$ and $\mathrm{DC}(\mathrm{N}=30)$ in 2012. There was no explicit spatial sampling scheme within each of these sites; these samples were simply used to make comparisons of yeast presence and density among sites. Only female-phase flowers (one flower per plant per sampling event) were collected to standardize comparisons 
across space and time. Third, to assess temporal variation across years, 40 plants per year were randomly bagged at DC at peak flower abundance from 2009-2012.

When interannual variation in yeast frequency of occurrence and abundance was sampled at DC from 2009-2012, we also wanted to assess whether any potential variation in yeast was associated with biotic factors, such as pollinator abundance, or abiotic factors. Bombus pollinator abundance was estimated by netting Bombus spp. near the DC site for $1 \mathrm{hr}$ each in the morning and afternoon during peak floral abundance. All Bombus species encountered (e.g., foraging on flowers, in flight, or on the ground) during sampling periods were netted, marked with an indelible dot of ink on their thorax to avoid recapturing the same individuals within sampling events, and released. Mean Bombus abundance was estimated as the catch rate (bees netted per hr) divided by the number of sampling events during the week of peak floral abundance (2009: 3 sampling events; 2010-2012: 7 sampling events in each year). As an abiotic factor that might be associated with interannual variation in yeast, we focused on snowpack. Delphinium initiates flowering in May with flowering extending through late June, and both the timing of flowering and flower production are strongly influenced by snowpack. In years with less snowpack, flowering is delayed and flower production is significantly lower likely due to colder temperatures and freezing events between the period between snowmelt and flowering (Inouye and McGuire, 1991). Lower snowpack is also associated with drier conditions (Pilon et al., 1994; Overpeck and Udall, 2010), and drought has been associated with reduced floral yeast in one other system (Golonka and Vilgalys, 2013). To examine the association between mean yeast and snowpack, we utilized measures of snowpack $(\mathrm{cm})$ on the ground as of May 1st in each year (2009-2012; data from billy barr, www.gothicwx.org). 


\section{Yeast occurrence and identification}

To determine yeast presence and density, nectar was extracted from flowers with sterile, calibrated microcapillary tubes and the volume of each sample was recorded (samples typically contained $<1 \mu 1$ of nectar). Each sample was diluted with 5-9 $\mu 1$ of $30 \%$ lactophenol cotton blue solution to facilitate microscopic visualization of yeasts. Cell density (cells $\mu \mathrm{l}^{-1}$ of nectar) was determined using a Neubauer chamber (Hausser Scientific, Horsham, PA, USA) and standard cell counting methods at a magnification of 400x (Nikon Eclipse E400, Melville, NY, USA) (akin to Herrera et al., 2008, 2009). Cells were identified as yeasts based on size and morphological features such as the presence of budding cells and large vacuoles, which contain refractive corpuscles. These density estimates integrate across all potential yeast taxa that are present in a sample, as it is difficult to determine species identity based on cell morphology and the staining solution kills the yeast. However, this coarse level of identification is suitable to address spatio-temporal variation in total yeast density and has been successfully used in other yeast surveys (Herrera et al., 2008, 2009).

Yeast identity was determined by removing nectar from a subset of Delphinium flowers $(\mathrm{N}=36$ flowers, each from a different plant) collected from BC on May 28, 2013 and streaking samples onto yeast malt agar (YM; Sigma-Aldrich, Saint Louis, MO, USA) supplemented with chloramphenicol. Including chloramphenicol reduced bacterial growth so that we could focus on yeast; assessing nectar bacterial abundance and diversity was beyond the scope of this study but can be assessed in future research. For each plate, one colony was picked for each morphologically distinct colony type and subsequently plated for further purification of isolates. Morphpotypes were distinguished by examining the relative size, hue, margin type, 3dimensional morphology, and opacity of colonies. DNA from isolates was extracted and 
amplified using a Sigma REDExtract-N-Amp Tissue polymerase chain reaction (PCR) kit (Sigma-Aldrich, Inc., Saint Louis, MO, USA). PCR reactions were performed in a volume of 20 $\mu \mathrm{l}$ using $0.8 \mu \mathrm{l}$ of extracted DNA, $10 \mu \mathrm{l}$ of REDExtract-N-Amp PCR Reaction Mix, $0.15 \mu \mathrm{l}$ of each primer at $50 \mu \mathrm{M}$ and $8.9 \mu 1$ of $\mathrm{H}_{2} \mathrm{O}$. A region of the D1/D2 domains of the large subunit of the nuclear ribosomal RNA gene was amplified using the primers NL1 and NL4 (Kurtzman and Robnett, 1998). PCR amplification was performed using a DNAEngine Thermal Cycler (Bio Rad Laboratories, Hercules, CA) using a touchdown PCR protocol outlined in Belisle et al. (2012). PCR products were separated by gel electrophoresis using $2 \%$ TBE agarose gel and visualized using ethidium bromide staining and subsequent UV transillumination (UVP Biosystems, Upland, CA). Samples that produced a visible band were sequenced on an ABI3730 Genetic Analyzer (Applied Biosystems, Carlsbad, CA) in one direction using the primer NL1. All sequences were trimmed and edited by eye using the program Sequencher v4.6 (Gene Codes, Corp., Ann Arbor, MI) and sequences were aligned to identify sequence polymorphisms. Operational taxonomic units (OTUs) were classified based on $\geq 98 \%$ similarity and queried against the entire nucleotide database (nr/nt) on GenBank for species identification using the Basic Local Alignment Search Tool (BLAST) (Kurtzman and Robnett, 1998). Representative sequences were deposited in GenBank (KJ473424 - KJ473425).

\section{Nectar chemistry}

To assess the correlation between yeast cell density and Delphinium nectar chemistry, nectar samples obtained from female-phase flowers collected at BC in 2011 on June $10(\mathrm{~N}=23$ flowers, each collected from a different plant) were analysed. To estimate fructose, glucose, and sucrose concentrations, the protocols of Vannette, Gauthier, and Fukami (2013) and Vannette 
and Fukami (2014) were followed. Briefly, a known volume of nectar was diluted in $100 \mu l$ of 50:50 acetonitrile:water containing $0.5 \mathrm{mg} \mathrm{ml}^{-1}$ maltose (Sigma-Aldrich, St. Louis, MO, USA) as an internal standard. Mono- and di-saccharide sugars were separated by Ultra Performance Liquid Chromatography (UPLC; Waters, Milford, MA, USA) on a Luna amide column (50 x 2 mm, $3 \mu \mathrm{m}$, Phenomenex, Torrance, CA, USA). An acetonitrile:water mobile phase with a 4.5 min linear gradient beginning at 80:20 MeCN:H2O and ending at 30:70 MeCN:H2O was used, with a 10 min equilibration at initial conditions between samples. Sugars were quantified using an evaporative light scattering (ELS) detector (Waters) and the concentration of each was calculated using the internal standard and a series of external standards. For each nectar sample analyzed, an associated sample partitioned from the original nectar source was analyzed for yeast cell density using the methods above to determine the association between cell density and nectar sugar concentrations.

\section{Statistical analyses}

Differences in both frequency of yeast occurrence and density across years (2009-2012 at DC) and among populations (BC, DC, and SC in 2012) were tested using Chi-square $\left(\chi^{2}\right)$ and Kruskal-Wallis tests, respectively. Homogeneity of variance was tested for amongst sampling years or populations using Levene's test for equality. To assess whether any potential interannual variation in yeast densities was associated with biotic or abiotic factors, we used Spearman rank correlations between mean yeast densities at DC from 2009 to 2012 with mean pollinator (Bombus) catch rate and snowpack for each year, respectively. To assess whether yeast density $(\log 10(\mathrm{x}+1)$-transformed $)$ varied across the flowering season at BC in 2012, a repeated-measures one-way ANOVA was performed. To test for differences in yeast cell density between flower 
sex phases, and whether the magnitude of difference varied amongst populations, a linear mixedeffects model was used with sex-phase and site as fixed effects and plant as a random effect. Cell density was $\log 10(\mathrm{x}+1)$ transformed prior to analysis to improve normality. Finally, Spearman rank correlations were used to test the significance of the association between yeast density $(\log 10(x+1)$-transformed $)$ and total sugar concentration, as well as the percent content of each nectar sugar component. All analyses were performed using R version 3.2.1 (R Core Development Team, 2011).

\section{Results}

\section{Frequency of occurrence and density of yeasts}

Virgin Delphinium flowers unvisited by pollinators did not harbor yeasts. In comparison, $60 \%$ of open flowers that were available to pollinators did harbor yeasts, providing support for the prediction that pollinators or other flower visitors are likely vectors for yeast inoculation of nectar.

At the temporal scale, there was no statistically significant variation across years (20092012) in the proportion of nectar samples colonized by yeasts and densities reached at DC during peak flowering (proportion of samples colonized: Chi-square $\chi^{2}=1.09, \mathrm{df}=3, P=0.90$; density: Kruskal-Walis $\left.\chi^{2}=6.911, \mathrm{df}=3, P=0.07\right)$. Across all years, the proportion of samples colonized by yeast at peak flowering ranged from $43-60 \%$, and mean yeast density per year ranged from $1.5 \times 10^{3}$ to $8.8 \times 10^{3}$ cells $\mu 1^{-1}$. Moreover, there was no significant heterogeneity of variances amongst years for yeast density estimates $\left(F_{3,153}=2.05, P=0.10\right)$. Furthermore, any subtle variation in mean yeast density among years did not correlate with either Bombus catch rate $\left(r_{s}=0.4, P=0.75\right)$ or snowpack $\left(r_{s}=-0.6, P=0.42\right)$. Bombus catch rate ranged from a low 
of 0.23 Bombus per hour in 2011 to a high of 3.38 Bombus per hour in 2010, and snowpack on May 1st ranged from $67 \mathrm{~cm}$ in 2009 to $0 \mathrm{~cm}$ in 2012 .

There was significant variation temporal variation in yeast cell densities within the flowering season at $\mathrm{BC}\left(F_{7,147}=5.77, P<0.0001\right)$. There were lower yeast cell densities at the start and end of the flowering season compared to the middle of the flowering season (Fig 1). Yeast densities were positively correlated with flower density, however this association was not significant $\left(r_{s}=0.67, \mathrm{~N}=8, P=0.08\right)$. At the scale of individual flowers, yeast density varied between floral sex phases $\left(F_{1,167}=45.65, P<0.0001\right)$, with female-phase flowers regularly harboring higher densities of yeasts $\left(6.67 \times 10^{3} \pm 1.55 \times 10^{3}\right.$ cells $\mu 1^{-1}$; mean \pm SE across all sites $)$ in comparison to those in male-phase $\left(4.23 \times 10^{2} \pm 1.36 \times 10^{2}\right.$ cells $\left.\mu \mathrm{l}^{-1}\right)$. The magnitude of this difference between floral-sex phases significantly varied across sites (Fig 2; site x phase interaction: $\left.F_{2,167}=4.68, P=0.01\right)$.

At the population scale, the proportion of samples colonized by yeasts varied significantly among Delphinium populations sampled in 2012 (Chi-Square $\chi^{2}=20.69, \mathrm{df}=2, P$ $<0.0001)$. BC had the highest proportion of samples colonized (77\%), while DC (52\%) and SC (61\%) had lower rates of colonization. Similarly, yeast densities varied significantly across Delphinium populations in 2012 (Kruskal-Wallis $\chi^{2}=52.9$, $\mathrm{df}=2, P<0.0001$ ), with BC having the highest average density $\left(4.0 \times 10^{4} \pm 6.7 \times 10^{3}\right.$ cells $\mu \mathrm{l}^{-1}$; interquartile range: $1 \times 10^{2}-3.6 \times$ $\left.10^{4}\right)$ in comparison to DC $\left(4.4 \times 10^{3} \pm 1.3 \times 10^{3}\right.$ cells $\mu 1^{-1}$; interquartile range: $\left.0-1.3 \times 10^{3}\right)$ and $\mathrm{SC}\left(3.5 \times 10^{3} \pm 7.7 \times 10^{2}\right.$ cells $\mu 1^{-1}$; interquartile range: $\left.0-3.7 \times 10^{3}\right)$. Finally, the variance of yeast density estimates significantly varied across $\operatorname{sites}\left(F_{2,365}=19.48, P<0.0001\right)$.

\section{Yeast identification}


A total of 54 isolates were obtained from the 36 nectar samples cultured. Fifty-three of the isolates were of a single species, the ascomycetous yeast Metschnikowia reukaufii (KJ473425). One isolate was the basidiomycete Cryptococcus saitoi (KJ473424).

\section{Nectar chemistry}

Nectar samples were sucrose-dominant, with sucrose being on average $80.7 \% \pm 4.3 \%$ ( \pm SE) of total sugar content. Total sugar concentration did not vary significantly with yeast density $\left(r_{s}=\right.$ $0.27, \mathrm{~N}=23, P=0.21)$; however, each individual nectar sugar component varied with yeast density (Fig 3). There was a significant negative correlation between sucrose concentration and yeast density $\left(r_{s}=-0.78, \mathrm{~N}=23, P<0.0001\right)$, whereas both fructose $\left(r_{s}=0.72, \mathrm{~N}=23, P=\right.$ $0.0001)$ and glucose $\left(r_{s}=0.60, \mathrm{~N}=23, P=0.003\right)$ percentages were positively correlated with increasing yeast density.

\section{Discussion}

Yeasts are frequent colonists of Delphinium nectar, occurring in samples obtained from all populations and across all years. We observed variation in yeast frequency of occurrence and density across multiple spatial and temporal scales, including across the populations and flowering season. These results corroborate previous nectar surveys demonstrating the commonality of yeasts as inhabitants of floral nectar (Brysch-Herzberg, 2004; Herrera et al., 2008, 2009; Belisle et al., 2012). Similarly, the frequency of occurrence and densities observed among samples fell within the range found in previous studies on the ecology and dynamics of nectar yeasts. For example, cell densities in the order of $10^{2}-10^{4}$ cells $\mu \mathrm{l}^{-1}$ were common among Delphinium nectar samples, matching levels observed in a number of other plant species on 
which nectar surveys have been conducted (Brysch-Herzberg, 2004; Herrera et al., 2009; Pozo et al., 2011; Canto and Herrera, 2012). Finally, culture-based techniques and molecular analysis revealed that nearly all isolates obtained from the subset of Delphinium nectar samples were Metschnikowia reukaufii, an ascomycetous yeast commonly found associated with floral nectar and animal visitors to flowers (Lachance et al., 2001).

Previous research has shown that yeast communities in nectar samples are species-poor, with usually only one or two species present in a nectar sample (Pozo et al., 2011, 2012). For example, Pozo et al. (2011) found an average species richness of 1.2 yeast species per nectar sample in their characterization of yeast communities associated with plant species in southern Spain. Moreover, over three quarters of the isolates obtained belonged to one of two Metschnikowia species (Pozo et al., 2011). Metschnikowia reukaufii similarly dominated nectar samples collected from Delphinium, with the only other yeast species identified in our sampling, Cryptococcus saitoi, represented by a single isolate. Prior research indicates that this yeast may not tolerate nectar conditions as well as M. reukaufii (Herrera et al., 2010). We note, however, that additional sampling of Delphinium nectar may reveal additional yeast species, as our sampling of a single population in one year may not capture the full extent of yeast diversity in this system.

At least three non-mutually exclusive mechanisms could explain low yeast species richness observed in Delphinium, as well as other plant species. The chemical characteristics of nectar, a function of both the host and its interaction with the environment, can act as an ecological filter, selecting for only a subset of yeast species that are dispersed to a flower. For instance, living in a nectar sugar environment creates high osmotic stress for yeast, which may lower the pool of potential yeast species that can survive in nectar. The sugar concentration of 
Delphinium nectar can exceed 50\% (Waser, 1978), and such high sugar concentrations may filter the species of yeast that can survive and proliferate in nectar unless they have physiological traits that allow them to cope with such high osmotic stress (Tokuoka, 1993; Herrera et al., 2010). Metschnikowia reukaufii can grow on media with 50\% sugar (glucose) concentration (Barnett et al., 2000; Pozo et al., 2012), suggesting the ability of this yeast to be able to survive and grow in the sugar concentration environment of Delphinium nectar. In addition, secondary metabolites and other compounds naturally found in nectar may have antimicrobial properties (Adler, 2000; Carter and Thornburg, 2004), and could reduce yeast species richness in nectar. Delphinium nectar contains low concentrations of norditerpene alkaloids (Cook et al., 2013), which are toxic to mammals and insects (Jennings et al., 1986; Manners et al., 1993; Manners et al., 1995) and may also reduce microbial growth in nectar. Other studies have shown variable effects of nectar secondary metabolites on yeast survival and growth, ranging from no effect to negative effects (Manson et al., 2007; Pozo et al., 2012). The effects of norditerpene alkaloids on yeast survival and growth warrant further investigation. Finally, priority effects generated through microbialinduced changes in nectar chemical components can exert a strong influence on community composition (Peay et al., 2012; Vannette and Fukami, 2014).

Though common, yeast abundance and frequency of occurrence varied both spatially and temporally. Significant spatial variation in both measures was detected amongst the three Delphinium populations sampled in 2012. Higher yeast densities and frequency of occurrence were observed at BC, while DC and SC were significantly lower. Variation in yeast abundance and frequency of occurrence may potentially be a function of differences in pollinator availability and rates of visitation across sites. Results from our survey, in addition to others, have revealed that pollinators and other floral visitors are likely the primary vectors of yeast in 
plant communities (Brysch-Herzberg, 2004; Herrera et al., 2009, 2010; Belisle et al., 2012).

Moreover, links have been observed between yeast abundance and frequency of occurrence with pollinator identity. For example, Herrera et al. (2009) and Pozo et al. (2014) found that bumble bee abundance and visitation to plants sampled in the Cazorla area of Spain are strong predictors of yeast abundance and density. Delphinium is reliant upon queen bumble bees, in addition to hummingbirds, for successful pollination (Waser, 1978). Fluctuations across populations in the timing of queen bumble bee emergence, arrival of migrant hummingbirds, their densities, and patterns of foraging activity may generate considerable variation in the dispersal dynamics of yeasts in these communities.

Temporal variation in yeast abundance and frequency was observed across some but not all of the scales examined, including flower lifetime and within a flowering season but not across years. Female-phase Delphinium flowers possessed significantly higher densities of yeasts in their nectar in comparison to younger, male-phase flowers. Similarly, a greater proportion of female-phase nectar samples contained yeasts, most notably at BC. Whether these differences were driven by differences in flower density or patterns of pollinator visitation amongst sites remains to be tested. These patterns match findings from other studies documenting temporal variation in frequency of occurrence and densities achieved with flower lifetime in hermaphroditic species (Herrera et al., 2008; Peay et al., 2012). However, this result is in contrast to findings in the dioecious plant Silene latifolia, where no difference was observed between the sexes (Golonka and Vilgalys, 2013). Such differences in findings among studies are likely a consequence of both duration of exposure to pollinators who vector yeasts, thus increasing the probability of inoculation, and incubation time. We also found that the proportion of nectar samples occupied by yeasts and densities observed varied significantly within the 
flowering season, being low at the start and end of the flowering season, and peaking during peak floral abundance in the middle of the blooming period. These findings match patterns observed in Helleborus foetidus (Pozo et al., 2014) and lend support to the notion that the ability of yeast to reach suitable habitat (i.e., nectar) and proliferate is likely limited at the beginning and end of the flowering season by pollinator activity. Pollinator visitation rates vary throughout a plant's flowering season, and studies have shown that peak pollinator visitation rates often correlate with peak floral abundance such that per-flower pollinator visitation rates are lower at the beginning and ends of flowering seasons (Thomson, 1982; Hegland and Totland, 2005). However, we cannot rule out the possibility that within-season variation in yeast is also driven by other biotic factors or abiotic conditions (e.g., Golonka and Vilgalys, 2013). It is important to note that although we found wide variation in Bombus catch rates and snowpack from 2009 to 2012, we found no significant interannual variation in yeast at peak floral abundance. Measuring how potential biotic and abiotic factors affect yeast frequency of occurrence and density within seasons will require not only correlational studies but also experiments to tease apart mechanisms.

Variation in the proportion of sugars in nectar was significantly correlated with yeast density. The relative abundance of sucrose decreased with increasing yeast density, while both that of fructose and glucose increased. These changes are likely a consequence of both pollinator visitation (Canto et al., 2008), yeast vectoring, and resulting microbial hydrolysis of the disaccharide sucrose (Phaff et al., 1978; Herrera et al., 2008). Such changes in the proportions of sucrose, fructose, and glucose have been observed in other systems (Herrera et al., 2008; Canto and Herrera, 2012), lending support to the hypothesis that microorganisms can leave a significant imprint on patterns of variation observed in nectar sugar components (Canto and Herrera, 2012). 
Nonetheless, plants also exhibit genetic variation in nectar rewards (Mitchell, 2004), and our approach limited our ability to tease apart the relative contribution of plant genotype vs. nectar microbiotia to variation in nectar sugar composition. Population genetic studies that use sterilized nectar to account for these factors will yield additional insights.

Intra- and inter-specific variation in nectar composition as a consequence of microbial metabolic activity may have important implications for pollinator foraging behavior and plant fitness, as both pollinator attraction and resulting plant fitness can be susceptible to changes in nectar sugar composition, concentration, and production (Wykes, 1952; Real and Rathcke, 1991; Mommaerts et al., 2013). Indeed, recent studies have demonstrated that microbial-induced changes in nectar can influence pollinator foraging behavior with significant consequences for plant fitness (Herrera et al., 2013; Vannette et al., 2013; Schaeffer and Irwin, 2014). For Delphinium, bumble bee pollinators respond positively to the presence of yeasts in nectar (Schaeffer and Irwin, 2014). This result is surprising considering that we documented here that yeast infested flowers had lower sucrose relative to glucose and fructose, and bees typically prefer sucrose-rich nectars (Mommaerts et al., 2013). Why free-flying bumble bee pollinators respond positively to the presence of yeast is unknown, although two relevant hypotheses are that: (1) yeasts are associated with scent cues that provide an honest to the presence of nectar rewards, and (2) pollinators seek out nectar yeasts as a dietary protein supplement. These and other hypotheses warrant experimental investigation. Though yeasts were sought by foraging bumble bees of Delphinium and H. foetidus (Herrera et al., 2013), effects on plant fitness varied depending on the component of plant reproduction considered. Yeasts enhanced male plant fitness, as measured through pollen donation, in Delphinium (Schaeffer and Irwin, 2014), whereas numerous estimates of female fitness, including number of pollen tubes in the style, fruit 
set, seed set, and seed mass, were all negatively affected by the presence of yeasts in $H$. foetidus (Herrera et al., 2013). Yeast effects on pollinator foraging behavior and plant fitness may not be universal, however. Vannette et al. (2013) found no direct effect of their presence or activity on measures of female fitness in the hummingbird-pollinated Mimulus aurantiacus, whereas Golonka (2002) observed marginally positive effects in Silene latifolia. Further research on the role of yeast as mediators of plant-pollinator interactions in other systems is needed to determine the degree of generality of these divergent effects.

In conclusion, our results document the ubiquity of yeasts in populations of a hermaphroditic perennial, in addition to highlighting how their dynamics can vary extensively on multiple spatio-temporal scales. Future studies are needed to assess how abiotic factors, plant traits, and species interactions interact to affect spatio-temporal dynamics in yeast density and diversity as well as the importance of this variation for pollination mutualisms.

\section{Acknowledgements}

The authors thank M. Bischoff, Z. Gezon, W. Kunkel, and A. Mapes for field assistance, R. Calsbeek, M. Duryea, and T. Fukami for lab support, and K. Alexander for access to lab facilities at Western State Colorado Univ. T. Fukami, R. Raguso, and four anonymous reviewers provided helpful comments on earlier versions of this manuscript. This work was supported by the Botanical Society of America Graduate Student Research Award, Dartmouth College Cramer Fund, and RMBL Dr. Lee R.G. Snyder and Dr. Jean H. Langenheim Graduate Fellowships awarded to RS. Additional support was provided by NSF grants awarded to RI (DEB-0841862 and DEB-0922080) and RI and RS (DEB-1311156). RV was supported by a Gordon and Betty Moore Foundation Fellowship from the Life Sciences Research Foundation. Any opinions, 
findings, and conclusions or recommendations expressed in this material are those of the authors and do not necessarily reflect the views of the National Science Foundation.

\section{References}

Adler, L. S., 2000. The ecological significance of toxic nectar. Oikos 91, 409-420.

Álvarez-Pérez, S., Herrera, C. M., de Vega, C., 2012. Zooming-in on floral nectar: a first exploration of nectar-associated bacteria in wild plant communities. FEMS Microbiology Ecology 80, 591-602.

Baker, H. G., Baker, I., 1973. Amino acids in nectar and their evolutionary significance. Nature 241, 543-545.

Baker, H. G., Baker, I. 1983. Floral nectar sugar constituents in relation to pollinator type. In: Jones, C. E. , Little, R. J. (eds.) Handbook of experimental pollination biology. New York: Van Nostrand Reinhold.

Baker, H. G., Baker, I., 1987. The Predictability of pollinator type by the chemistry of nectar. American Journal of Botany 74, 645-645.

Barnett, J., Payne, R., Yarrow, D. 2000. Yeasts: characteristics and identification, Cambridge, U.K., Cambridge University Press.

Belisle, M., Peay, K., Fukami, T., 2012. Flowers as islands: spatial distribution of nectarinhabiting microfungi among plants of Mimulus aurantiacus, a hummingbird-pollinated shrub. Microbial Ecology 63, 711-718.

Bosch, M., Waser, N. M., 1999. Effects of local density on pollination and reproduction in Delphinium nuttallianum and Aconitum columbianum (Ranunculaceae). American Journal of Botany 86, 871-879.

Brysch-Herzberg, M., 2004. Ecology of yeasts in plant-bumblebee mutualism in Central Europe. FEMS Microbiology Ecology 50, 87-100.

Canto, A., Herrera, C. M., 2012. Micro-organisms behind the pollination scenes: microbial imprint on floral nectar sugar variation in a tropical plant community. Annals of Botany $110,1173-1183$.

Canto, A., Herrera, C. M., Medrano, M., Pérez, R., García, I. M., 2008. Pollinator foraging modifies nectar sugar composition in Helleborus foetidus (Ranunculaceae): An experimental test. American Journal of Botany 95, 315-320.

Carter, C., Thornburg, R. W., 2004. Is the nectar redox cycle a floral defense against microbial attack? Trends in Plant Science 9, 320-324.

Cook, D., Manson, J. S., Gardner, D. R., Welch, K. D., Irwin, R. E., 2013. Norditerpene alkaloid concentrations in tissues and floral rewards of larkspurs and impacts on pollinators. Biochemical Systematics and Ecology 48, 123-131.

de Vega, C., Herrera, C. M., 2012. Relationships among nectar-dwelling yeasts, flowers and ants: patterns and incidence on nectar traits. Oikos 121, 1878-1888.

Fridman, S., Izhaki, I., Gerchman, Y., Halpern, M., 2012. Bacterial communities in floral nectar. Environmental Microbiology Reports 4, 97-104.

Golonka, A. M., 2002. Nectar-inhabiting microorganisms (NIMs) and the dioecious plant species Silene latifolia. Ph.D. Dissertation. Duke University, Durham, N.C. 
Golonka, A. M., Johnson, B. O., Freeman, J., Hinson, D. W., 2014. Impact of nectarivorous yeasts on Silene caroliniana's scent. Eastern Biologist 3, 1-26.

Golonka, A. M., Vilgalys, R., 2013. Nectar inhabiting yeasts in Virginian populations of Silene latifolia (Caryophyllaceae) and coflowering species. The American Midland Naturalist 169, 235-258.

Goodrich, K. R., Zjhra, M. L., Ley, C. A., Raguso, R. A., 2006. When flowers smell fermented: The chemistry and ontogeny of yeasty floral scent in pawpaw (Asimina triloba: Annonaceae). International Journal of Plant Sciences 167, 33-46.

Hegland, S. J., Totland, Ø., 2005. Relationships between species' floral traits and pollinator visitation in a temperate grassland. Oecologia 145, 586-594.

Herrera, C. M., Canto, A., Pozo, M. I., Bazaga, P., 2010. Inhospitable sweetness: nectar filtering of pollinator-borne inocula leads to impoverished, phylogenetically clustered yeast communities. Proceedings of the Royal Society B: Biological Sciences 277, 747-754.

Herrera, C. M., de Vega, C., Canto, A., Pozo, M. I., 2009. Yeasts in floral nectar: a quantitative survey. Annals of Botany 103, 1415-1423.

Herrera, C. M., García, I. M., Pérez, R., 2008. Invisible floral larcenies: microbial communities degrade floral nectar of bumble bee-pollinated plants. Ecology 89, 2369-2376.

Herrera, C. M., Pozo, M. I., 2010. Nectar yeasts warm the flowers of a winter-blooming plant. Proceedings of the Royal Society of London B: Biological Sciences 277, 1827-1834.

Herrera, C. M., Pozo, M. I., Mendrano, M., 2013. Yeasts in nectar of an early-blooming herb: sought by bumble bees, detrimental to plant fecundity. Ecology 94, 273-279.

Inouye, D. W., McGuire, A. D., 1991. Effects of snowpack on timing and abundance of flowering in Delphinium nelsonii (Ranunculaceae): implications for climate change. American Journal of Botany 78, 997-1001.

Jennings, K. R., Brown, D. G., Wright, D. P., Jr., 1986. Methyllycaconitine, a naturally occurring insecticide with a high affinity for the insect cholinergic receptor. Experientia 42, 611613.

Kevan, P. G., Eisikowitch, D., Fowle, S., Thomas, K., 1988. Yeast-contaminated nectar and its effects on bee foraging. Journal of Apicultural Research 27, 26-29.

Kurtzman, C. P., Robnett, C. J., 1998. Identification and phylogeny of ascomycetous yeasts from analysis of nuclear large subunit 26S ribosomal DNA partial sequences. Antonie Van Leeuwenhoek International Journal of General and Molecular Microbiology 73, 331371.

Lachance, M.-A., Starmer, W. T., Rosa, C. A., Bowles, J. M., Barker, J. S. F., Janzen, D. H., 2001. Biogeography of the yeasts of ephemeral flowers and their insects. FEMS Yeast Research 1, 1-8.

Manners, G. D., Panter, K. E., Pelletier, S. W., 1995. Structure-activity relationships of norditerpenoid alkaloids occurring in toxic larkspur (Delphinium) species. Journal of Natural Products 58, 863-869.

Manners, G. D., Panter, K. E., Ralphs, M. H., Pfister, J. A., Olsen, J. D., James, L. F., 1993. Toxicity and chemical phenology of norditerpenoid alkaloids in the tall larkspurs (Delphinium species). Journal of Agricultural and Food Chemistry 41, 96-100.

Manson, J., Lachance, M.-A., Thomson, J., 2007. Candida gelsemii sp. nov., a yeast of the Metschnikowiaceae clade isolated from nectar of the poisonous Carolina jessamine. Antonie van Leeuwenhoek 92, 37-42. 
Mitchell, R. J., 2004. Heritability of nectar traits: why do we know so little? Ecology 85, 15271533.

Mommaerts, V., Wäckers, F., Smagghe, G., 2013. Assessment of gustatory responses to different sugars in harnessed and free-moving bumblebee workers (Bombus terrestris). Chemical Senses, bj014.

Overpeck, J., Udall, B., 2010. Dry times ahead. Science 328, 1642-1643.

Peay, K. G., Belisle, M., Fukami, T., 2012. Phylogenetic relatedness predicts priority effects in nectar yeast communities. Proceedings of the Royal Society B: Biological Sciences 279, 749-758.

Phaff, H. J., Miller, M. W., Mrak, E. M. 1978. The life of yeasts, Cambridge, Massachusetts, Harvard University Press.

Pilon, C. E., Côté, B., Fyles, J. W., 1994. Effect of snow removal on leaf water potential, soil moisture, leaf and soil nutrient status and leaf peroxidase activity of sugar maple. Plant and Soil 162, 81-88.

Pleasants, J., Zimmerman, M., 1979. Patchiness in the dispersion of nectar resources: Evidence for hot and cold spots. Oecologia 41, 283-288.

Pozo, M., Herrera, C.M., Bazaga, P., 2011. Species richness of yeast communities in floral nectar of southern Spanish plants. Microbial Ecology 61, 82-91.

Pozo, M., Herrera, C. M., Alonso, C., 2014. Spatial and temporal distribution patterns of nectarinhabiting yeasts: how different floral microenvironments arise in winter-blooming Helleborus foetidus. Fungal Ecology 11, 173-180.

Pozo, M. I., Lachance, M.-A., Herrera, C. M., 2012. Nectar yeasts of two southern Spanish plants: the roles of immigration and physiological traits in community assembly. FEMS Microbiology Ecology 80, 281-293.

Price, M. V., Waser, N. M., 1979. Pollen dispersal and optimal outcrossing in Delphinium nelsonii. Nature 277, 294-297.

R Core Development Team, 2011. R: A language and environment for statistical computing. In. R Foundation for Statistical Computing Vienna, Austria.

Raguso, R. A., 2004. Why are some floral nectars scented? Ecology 85, 1486-1494.

Real, L. A., Rathcke, B. J., 1991. Individual variation in nectar production and its effects on fitness in Kalmia latifolia. Ecology 72, 149-155.

Schaeffer, R. N., Irwin, R. E., 2014. Yeasts in nectar enhance male fitness in a montane perennial herb. Ecology 95, 1792-1798.

Simpson, B. B., Neff, J. L. 1983. Evolution and diversity of floral rewards. In: Jones, C. E. , Little, R. J. (eds.) Handbook of experimental pollination biology. New York: Van Nostrand Reinhold.

Thomson, J. D., 1982. Patterns of visitation by animal pollinators. Oikos 39, 241-250.

Tokuoka, K., 1993. Sugar- and salt-tolerant yeasts. Journal of Applied Bacteriology 74, 101-110.

Vannette, R. L., Fukami, T., 2014. Historical contingency in species interactions: towards nichebased predictions. Ecology Letters 17, 115-124.

Vannette, R. L., Gauthier, M.-P. L., Fukami, T., 2013. Nectar bacteria, but not yeast, weaken a plant-pollinator mutualism. Proceedings of the Royal Society B: Biological Sciences 280, 20122601.

Waser, N. M., 1978. Competition for hummingbird pollination and sequential flowering in two Colorado wildflowers. Ecology 59, 934-944. 
Waser, N. M., Mitchell, R. J., 1990. Nectar standing crops in Delphinium nelsonii flowers: spatial autocorrelation among plants? Ecology 71, 116-123.

Waser, N. M., Price, M. V., 1981. Pollinator choice and stabilizing selection for flower color in Delphinium nelsonii. Evolution 35, 376-390.

Waser, N. M., Price, M. V., 1991. Outcrossing distance effects in Delphinium nelsonii: pollen loads, pollen tubes, and seed set. Ecology 72, 171-179.

Wykes, G. R., 1952. The preferences of honey bees for solutions of various sugars which occur in nectar. Journal of Experimental Biology 29, 511-518.

\section{Figure legends}

Fig. 1. Temporal variation in yeast cell (black line) and flower (dashed line) density across the 2012 Delphinium nuttallianum flowering season at Brush Creek. Dots represent means and vertical segments are $\pm 1 \mathrm{SE}$. Note the different scales used for each y-axis.

Fig. 2. Yeast cell density in the nectar of male- and female-phase flowers obtained from three populations of Delphinium nuttallianum in 2012. Triangles and dots represent male- and femalephase means respectively. Vertical segments are $\pm 1 \mathrm{SE}$.

Fig. 3. Associations between proportion (A-C) of sucrose, fructose, and glucose and yeast cell density in single nectar samples of Delphinium nuttallianum. 


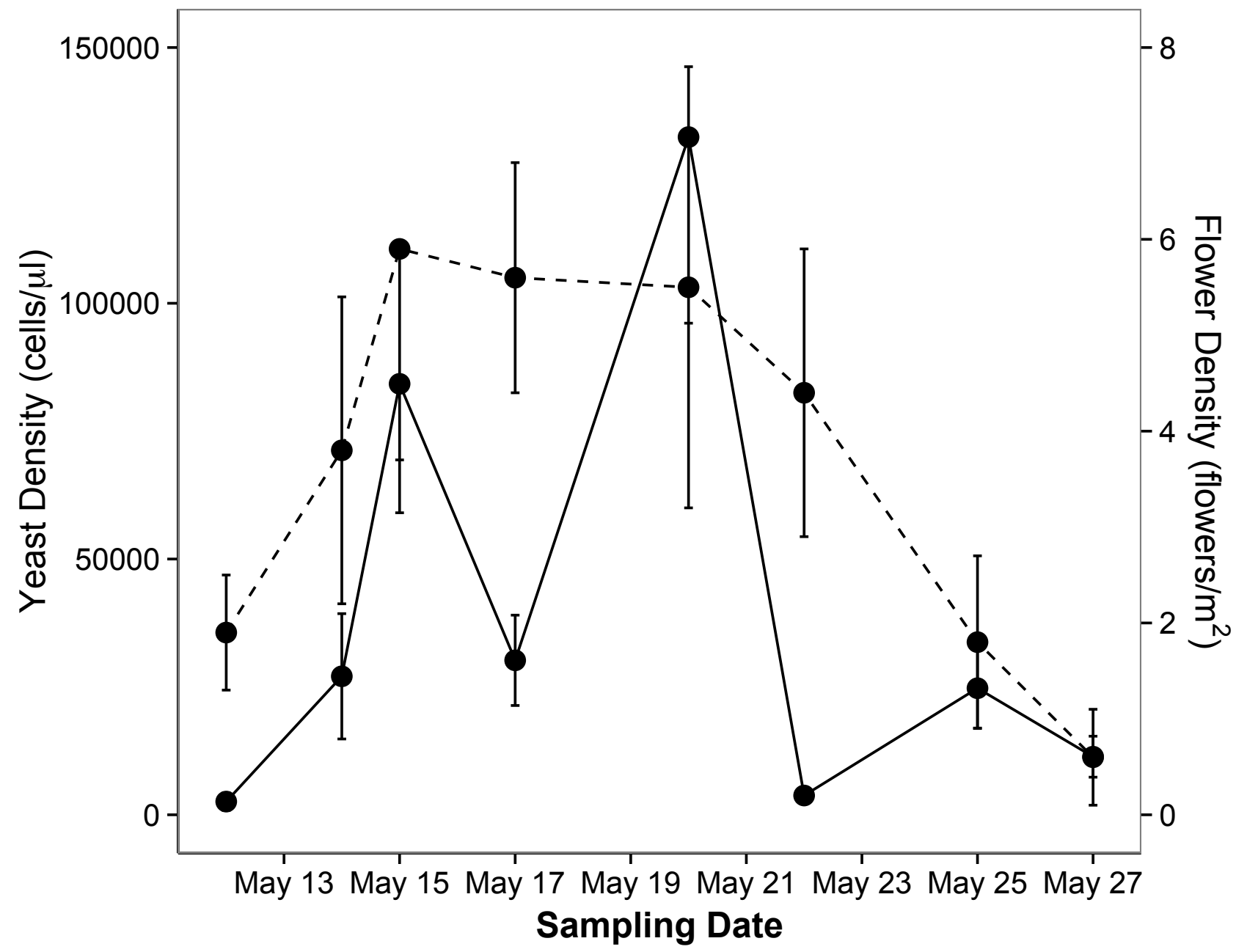


Figure 2

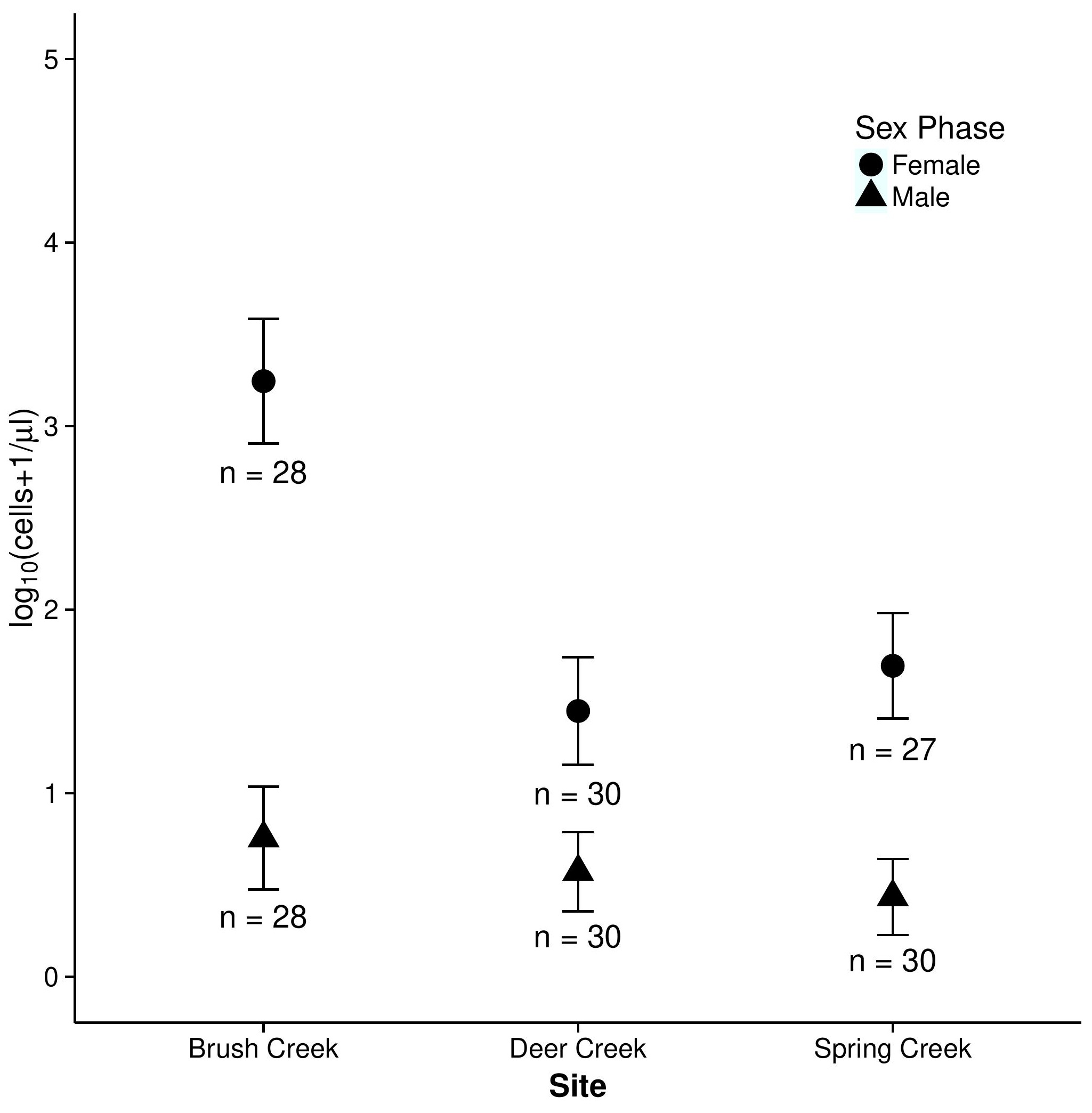



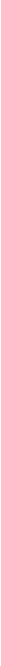

\section{Figure 3}

$\log _{10}$ (cell density)

\begin{abstract}
政
\end{abstract}
$\log _{10}$ (cell density) 